\title{
Acute Simultaneous Ruptures of the Anterior Cruciate Ligament and Patellar Tendon
}

\author{
Dong Hwi Kim, MD, Gwang Chul Lee, MD, and Sung-Hae Park, MD \\ Department of Orthopaedic Surgery, Chosun University Hospital, Gwangju, Korea
}

\begin{abstract}
Acute simultaneous rupture of the anterior cruciate ligament (ACL) and patellar tendon is a rare injury. We present a case report of a 32-year-old male patient with ruptured ACL and ipsilateral patellar tendon rupture sustained while playing baseball. Surgery was performed on the patellar tendon and the ACL simultaneously. The clinical and radiological outcomes of the treatment were successful. We present this case with a review of the literatures.
\end{abstract}

Keywords: Anterior cruciate ligament, Patellar tendon, Acute rupture, Repair

Anterior cruciate ligament (ACL) rupture is a relatively common sports injury, whereas patellar tendon rupture is rare. Acute simultaneous rupture of the ACL and patellar tendon has been addressed in only 1 case report domestically and approximately 20 reports internationally ${ }^{1-7)}$. Here, we report a case of a simultaneous rupture of the ACL and patellar tendon of the right knee sustained while playing baseball. The patellar tendon was repaired with a primary suture and the ACL was reconstructed simultaneously using an Achilles tendon allograft. The one-year follow-up outcomes were satisfactory.

\section{Case Report}

A 32-year-old male visited our clinic with a major complaint of severe pain in the right knee that started to occur when he managed to stop running over the second base that he had run toward while playing baseball. The right leg that was planted on

Received July 9, 2013; Revised (1st) October 4, 2013;

(2nd) November 29, 2013; Accepted December 2, 2013

Correspondence to: Dong Hwi Kim, MD

Department of Orthopaedic Surgery, Chosun University Hospital, 365

Pilmun-daero, Dong-gu, Gwangju 501-717, Korea

Tel: +82-62-220-3147, Fax: +82-62-226-3379

E-mail: osbaggu@hanmail.net

This is an Open Access article distributed under the terms of the Creative Commons Attribution Non-Commercial License (http://creativecommons.org/licenses/by-nc/3.0/) which permits unrestricted non-commercial use, distribution, and reproduction in any medium, provided the original work is properly cited. the second base at the time of injury produced a crackling sound when the knee was flexed with a valgus force applied and severe swelling of the right knee, range of motion (ROM) restriction due to pain, and approximately $40^{\circ}$ of extension lag were present. Simple plain radiography showed patella alta on the lateral view (Fig. 1), but other abnormal findings, such as a fracture, were not observed. Magnetic resonance imaging (MRI) was performed to identify if there is an accompanying injury, which revealed a complete tear of the patellar tendon and complete tears of the ACL and the medial collateral ligament (MCL) at the femoral

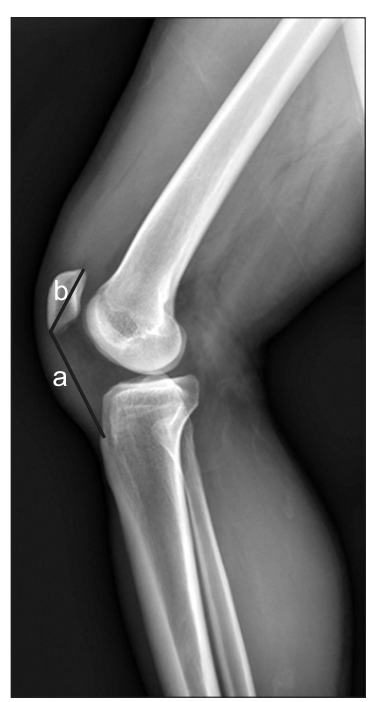

Fig. 1. Lateral radiograph of the right knee following the initial injury demonstrating swelling and patella alta (Insall-Salvati ratio $[\mathrm{a} / \mathrm{b}]=1.69$ ). 

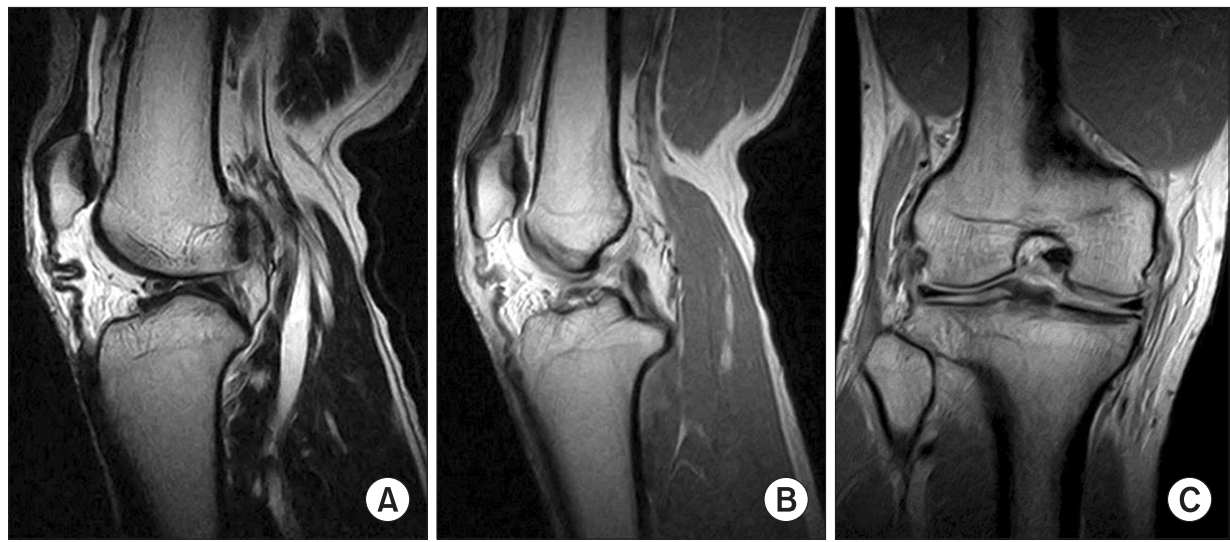

Fig. 2. Magnetic resonance imaging findings of the right knee following the initial injury. (A) T2-weighted sagittal image shows a rupture of the patellar tendon. (B) T2-weighted sagittal image demonstrates a complete tear of the anterior cruciate ligament at the femoral attachment site. (C) Coronal proton density image shows a medial collateral ligament rupture at the femoral attach site.
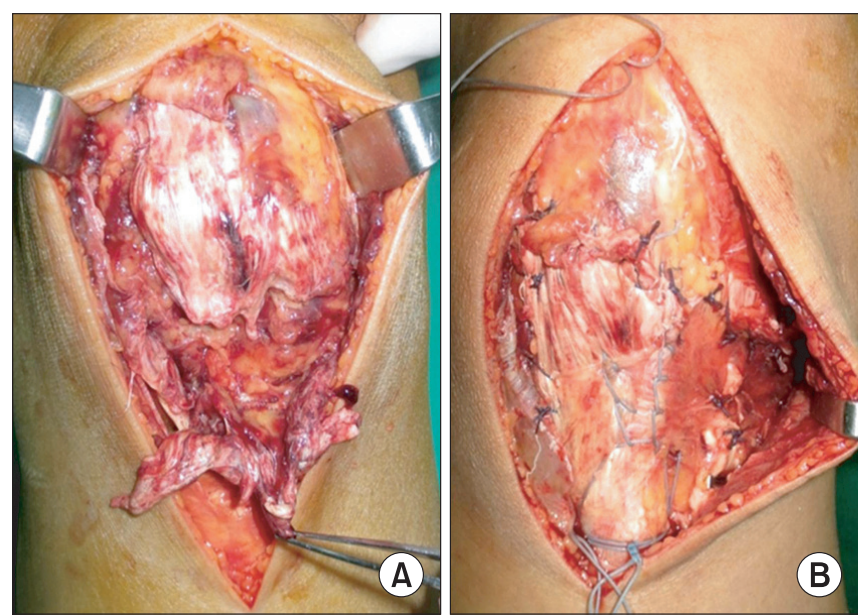

Fig. 3. (A) The intraoperative photograph shows that the patellar tendon is completely torn in the midsubstance and tear margins are frayed like a mop-end. (B) Three core sutures were done using the Krackow method in the patellar tendon into a bony tunnel with fiberwire.

attachment sites, and increased signal intensity in the lateral meniscus led us to suspect an injury to the meniscus (Fig. 2).

At 6 days after injury, primary suture repair of the patellar tendon with ACL reconstruction was carried out. The patellar tendon repair preceded the ACL reconstruction. Initially, a 10$\mathrm{cm}$ longitudinal skin incision extending from the middle of the patella through the center of the patellar tendon to the tibial tuberosity was made to expose the ruptured patellar tendon. The patellar tendon was completely torn in the midsubstance, the torn end had a frayed mop-end appearance, and the tear extended to the medial retinaculum (Fig. 3A). Taking consideration into the shape of the torn end and the distally narrowing anatomical shape of the patellar tendon, the torn end was longitudinally divided into three equal sections, and three core sutures of the patellar tendon were determined. The center of the distal end was repaired with a Fiberwire (Arthrex, Naples, FL, USA) suture using the Krackow method. Two longitudinal bone tunnels were created in the patella using a 2.0-mm Kirschner wire. A suture was passed through the tunnel and pullout repair with the proximal end was performed on the anterosuperior patella with the knee in $45^{\circ}$ flexion. The proximal end was sutured with two Fiberwire sutures at medial $1 / 3$ and lateral $1 / 3$ points using the Krackow method. A bone tunnel parallel to the tibial tuberosity was created, through which the sutures were passed and pullout suture with the distal torn end was done. At the torn ends, endto-end anastomosis was performed additionally (Fig. 3B). Subsequently, an anterolateral portal, an anteromedial portal, and an accessory far medial portal for anatomical ACL reconstruction were created through the skin incision. An arthroscope was introduced into the joint. The presence of a transverse tear in the posterior horn of the lateral meniscus that was not clearly identifiable on MRI was confirmed. It was repaired using an all inside technique with two PDS (polydioxanone monofilament; Ethicon Inc., Somerville, NJ, USA) sutures. A complete tear of the ACL at the femoral attachment site was confirmed, and reconstruction was done using an Achilles tendon allograft to avoid a donor site injury (Fig. 4). For anatomical ACL reconstruction, a femoral tunnel was created at the center of the femoral attachment of the ACL using the transportal technique and a tibial tunnel was made at the center of the tibial attachment site using Pinn-ACL tibial guide (Linvatec, Largo, FL, USA). With the calcaneal fragment of the Achilles tendon allograft placed toward the femur, the graft was fixated to the femur using a metal interference screw and to the tibia using a bioabsorbable interference screw with the knee in $20^{\circ}$ flexion, and a metal staple was used for additional fixation. Following ACL reconstruction, a primary suture for the medial retinaculum and the patellar tendon sheath was carried out. The complete tear of the MCL at the femoral attachment site was conservatively treated: after 2 weeks of splint immobilization, rehabilitation therapy was administered so that the range of flexion 

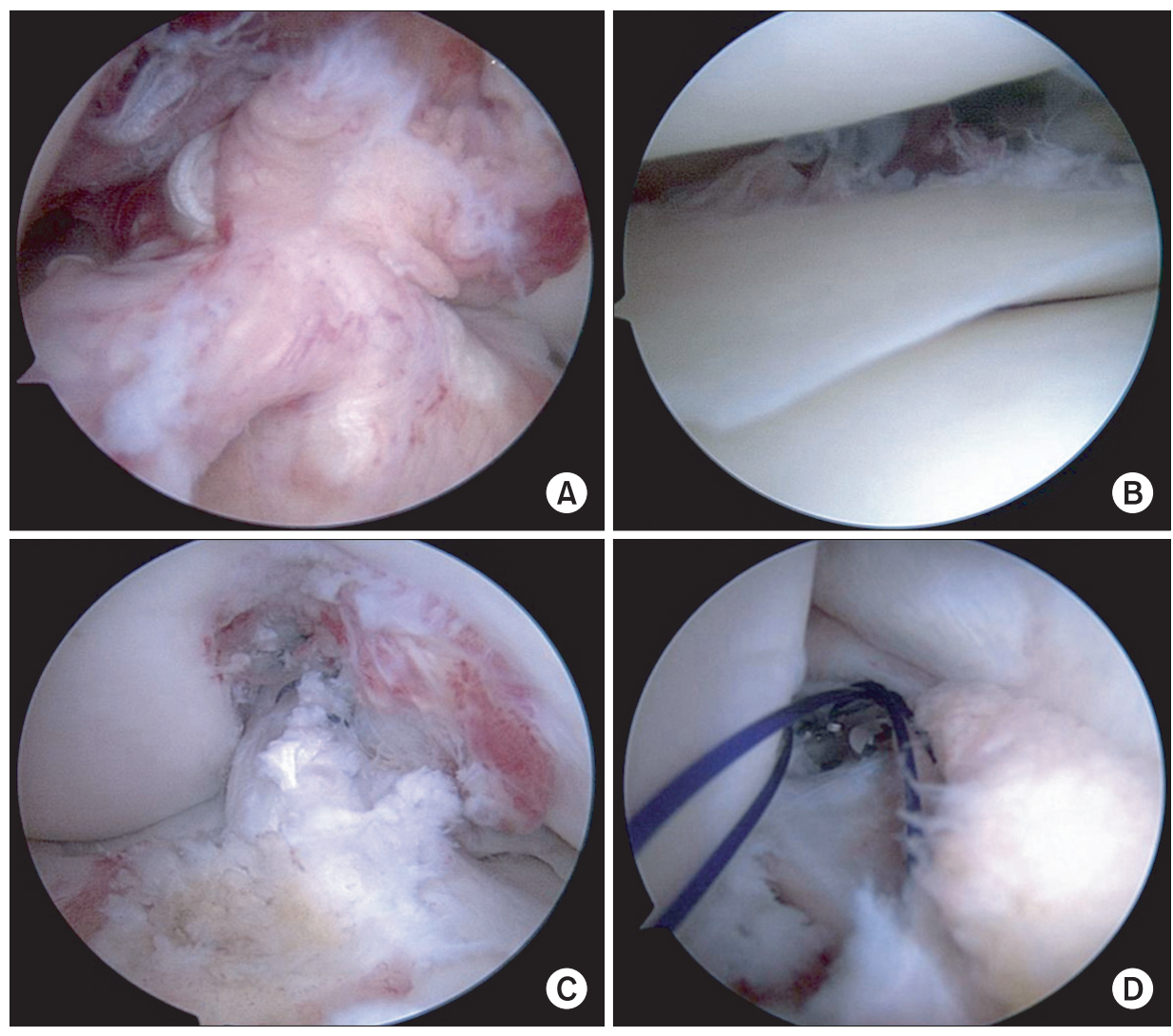

Fig. 4. Intraoperative Arthroscopy. (A) Torn anterior cruciate ligament (ACL). (B) Peripheral longitudinal tear of the posterior horn of the lateral meniscus. (C) Anatomical reconstruction of the ACL with an Achilles tendon allograft visualized from the anteromedial portal. (D) Repair of the lateral meniscus with PDS (violet 1).
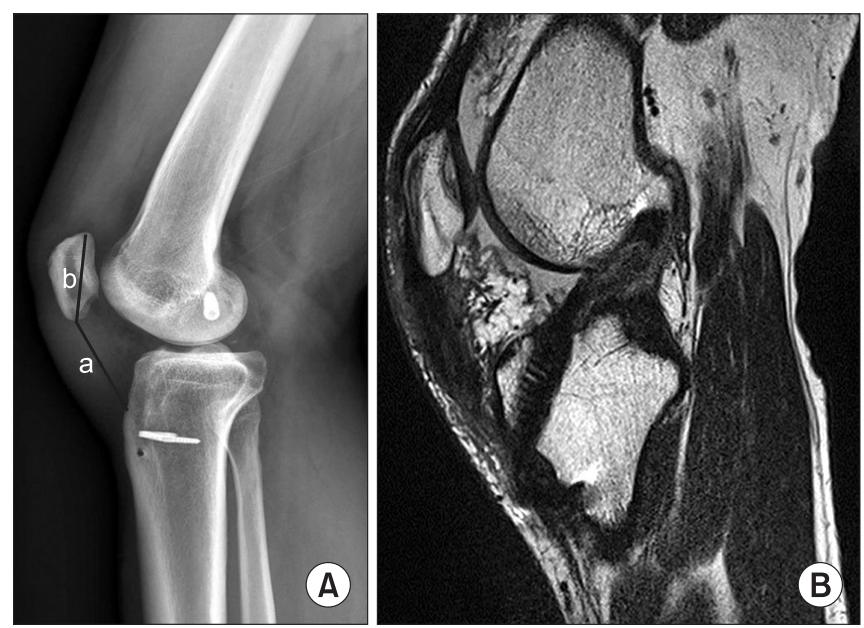

Fig. 5. (A) Lateral simple radiograph of the right knee at the last followup demonstrating proper position of the screws and appropriate position of the patella (Insall-Salvati ratio $[\mathrm{a} / \mathrm{b}]=1.15$ ). (B) T2-weighted sagittal image of the right knee at the last follow-up showing the continuity of the reconstructed anterior cruciate ligament and the patellar tendon.

could be gradually increased.

For the 14 postoperative days, the patient was treated with compression dressing, application of ice, and elevation of the leg with a long leg splint applied, to reduce swelling. From day
1 after surgery, quadriceps strengthening exercises were recommended. From the second postoperative week, the long leg splint was replaced with a limited motion knee brace and the patient was encouraged to perform flexion exercises within pain-free ranges. The range of flexion was gradually increased to $30^{\circ}$ at the 2nd postoperative week, to $60^{\circ}$ at the 4 th postoperative week, and to $90^{\circ}$ at the 6 th postoperative week. Weight bearing ambulation was not allowed until 6 weeks after surgery due to the meniscal repair.

MRI scan at 6 month after surgery showed well-maintained continuity of the ACL and patellar tendon and no abnormal findings in other soft tissue, such as the meniscus (Fig. 5). At 1-year postoperative follow-up, $0^{\circ}-140^{\circ}$ of flexion was possible, and no changes were detected in the Lachman and pivot shift tests. The Lysholm score was satisfactorily high (94 points). Other than discomfort in the knee after long or knee-straining activities, no complication was reported.

\section{Discussion}

Costa-Paz et al. ${ }^{4)}$ and Levakos et al. ${ }^{5)}$ reported that 7 cases of simulatenous rupture of the ACL and the patellar tendon were not identified in early diganosis due to the failure of detecting an 
ACL rupture in 4 cases and a patellar tendon rupture in 3 cases. MRI is the most important diagnostic tool. In our case, based on the radiographic evidence of patellar alta and physical examination finding of an extension lag, the initial diagnosis of a patellar tendon rupture was made. The combined ACL rupture and MCL rupture were difficult to detect during physical examination due to pain, but MRI was useful in the detection of the two combined injuries. Therefore, we suggest that when a patellar tendon injury is suspected, MRI should be performed to identify the presence of a combined injury. Intraoperatively, physical and arthroscopic examinations should be performed carefully with the patient under anesthesia to detect any undiagnosed injury.

The mechanism of injury of simultaneous rupture of the ACL and the patellar tendon has yet to be established. In general, it has been understood that it is related to rapid deceleration of the flexed knee on a fixed foot and eccentric contraction of the quadriceps $^{6-8)}$.

In our case, when the patient attempted not to overrun the second base, the right knee was flexed with the foot fixed on the ground and the upper body was tilted toward the outfield. This caused application of a tremendous valgus force and a rotational force on the knee and eccentric quadriceps contraction, resulting in a patellar tendon rupture and subsequently tears of the ACL and MCL. Therefore, in our opinion, sliding can be an important skill to prevent extensive damage to the knee in amateur baseball. In the treatment of acute simultaneous rupture of the ACL and the patellar tendon, a delayed patellar tendon repair can lead to extension limitation, persistent quadriceps atrophy, and scar tissue formation and degeneration at the torn end. Then, complete healing may become difficult to achieve and complications, such as patella alta, can occur ${ }^{6}$. In addition, the timing of patellar tendon repair is closely associated with the postoperative function of the knee ${ }^{9)}$. Thus, there is no disagreement on the need for prompt patellar tendon repair. However, it still remains controversial whether the concurrent ACL injury should be treated simultaneously or the ACL should reconstructed after sufficient period of rehabilitation following patellar tendon repair. In addition, agreement is elusive on the type of tendon graft for reconstruction and rehabilitation regimen.

Although we took consideration into a staged repair, simultaneous repair was determined necessary based on the following grounds: the simultaneous rupture was accompanied by a complete tear of the MCL, causing severe joint instability; the patient was relatively young; a staged repair would prolong the treatment period; and intra-articular lesions could remain undetected. During surgery, an acute longitudinal meniscal tear that was uniden- tified on MRI was found and treated. We believe if a staged repair had been our treatment of choice, the meniscal repair would have been delayed, compromising complete healing. However, simultaneous patellar tendon repair and ACL reconstruction carries the risk of causing additional damage to the already severely injured knee, resulting in joint stiffness, and the two procedures require different rehabilitation regimens. In our case, weightbearing ambulation was not allowed for 6 weeks after surgery to prevent joint stiffness, whereas knee flexion exercises were initiated relatively early at 2 weeks after surgery and the range of flexion was increased progressively. We believe that anatomic ACL reconstruction using an allograft contributed to reducing the operation time and thus minimizing iatrogenic trauma. In our patient, 3 core sutures and end-to-end anastomosis were performed simultaneously during the patellar tendon repair. Although the torn end had a frayed mop-end appearance, neither patellar alta related to quadriceps atrophy nor scar tissue formation at the torn end was present because the injury was in the acute stage, which facilitated the patellar tendon repair. As the sutures were considered firmly placed, joint exercises were started relatively early at 2 weeks after surgery, which also contributed to prevention of arthrofibrosis. Anatomical ACL reconstruction and additional simultaneous procedures for concomitant intra-articular lesions, such as the meniscal tear, helped reduce the treatment period.

In the absence of reports that provide guidelines for the timing of the patellar tendon and ACL repair, we carried out patellar tendon repair first before ACL reconstruction. By placing the superiorly translated patellar tendon back into the anatomical position during the preceding patellar tendon repair, arthroscopic portals could be established at exact locations and by preventing extravasation of saline that could have been unavoidable if arthroscopic surgery had preceded the patellar tendon repair, paratenon suture that facilitates patellar tendon healing could be performed during wound repair, and ACL reconstruction could be carried out without an additional incision.

The femoral tunnel could be created at an anatomical location during ACL reconstruction using a transportal technique. Although the tibial tunnel was created with the knee in approximately $120^{\circ}$ flexion, it appeared to have no influence on the patellar tendon suture site due to the relatively firm core sutures and end-to-end anastomosis.

Rehabilitation after patellar tendon repair is more conservative than that after ACL reconstruction. Thus, simultaneous repair requires a modified rehabilitation regimen. In general, an isolated patellar tendon tear repair is followed by the initiation of quad- 
riceps muscle strengthening exercises at 3 weeks after surgery and 6 weeks of cast immobilization in extension. At 6 weeks after surgery, the cast is replaced with a brace and up to $45^{\circ}$ of ROM is permitted. Afterwards, the ROM is increased by $10^{\circ}-15^{\circ}$ per week ${ }^{10)}$. In our patient, quadriceps strengthening exercises were allowed with a long leg splint worn on the 1st postoperative day, flexion/extension exercises were carried out with a limited motion knee brace worn from the 2 nd postoperative week. No complications, such as joint stiffness, were noted because of early rehabilitation.

Acute simultaneous rupture of the ACL and the patella tendon is a rare injury and early diagnosis requires careful physical examination and MRI. In the absence of agreed treatment protocols, we suggest that simultaneous repair of the patellar tendon and ACL reconstruction using an Achilles tendon allograft can be an effective treatment method that is advantageous in preventing joint stiffness through rapid rehabilitation and allowing prompt treatment of a concomitant intra-articular lesion.

\section{Conflict of Interest}

No potential conflict of interest relevant to this article was reported.

\section{Acknowledgements}

This study was supported by research fund from Chosun University Hospital, 2012.

\section{References}

1. Tsarouhas A, Iosifidis M, Kotzamitelos D, Traios S. Combined rupture of the patellar tendon, anterior cruciate ligament and lateral. Hippokratia. 2011;15:178-80.

2. Chiang AS, Shin SS, Jazrawi LM, Rose DJ. Simultaneous ipsilateral ruptures of the anterior cruciate ligament and patellar tendon: a case report. Bull Hosp Jt Dis. 2005;62:134-6.

3. Kim YJ, Kim TK, Yang HD, Kim HJ, Park JY, Seo KH. Simultaneous acute rupture of anterior cruciate ligament and patellar tendon. J Korean Arthrosc Soc. 2006;10:103-7.

4. Costa-Paz M, Muscolo DL, Makino A, Ayerza MA. Simultaneous acute rupture of the patellar tendon and the anterior cruciate ligament. Arthroscopy. 2005;21:1143.

5. Levakos Y, Sherman MF, Shelbourne KD, Trakru S, Bonamo JR. Simultaneous rupture of the anterior cruciate ligament and the patellar tendon: six case reports. Am J Sports Med. 1996;24:498-503.

6. Futch LA, Garth WP, Folsom GJ, Ogard WK. Acute rupture of the anterior cruciate ligament and patellar tendon in a collegiate athlete. Arthroscopy. 2007;23:112.e1-4.

7. Wissman RD, Vonfischer N, Kempf K. Acute concomitant anterior cruciate ligament and patellar tendon tears in a nondislocated knee. J Clin Imaging Sci. 2012;2:3.

8. Baker BE. O'Donoghue's triad plus patellar tendon rupture. N Y State J Med. 1980;80:1436-7.

9. Siwek CW, Rao JP. Ruptures of the extensor mechanism of the knee joint. J Bone Joint Surg Am. 1981;63:932-7.

10. Campbell WC, Canale ST, Beaty JH, eds. Campbell's operative orthopaedics. 11th ed. Philadelphia: Mosby; 2008. p2747-78. 